\title{
Oxide semiconductors for solar to chemical energy conversion: nanotechnology approach
}

\begin{abstract}
The present work considers the application of oxide semiconductors in the conversion of solar energy into the chemical energy required for water purification (removal of microbial cells and toxic organic compounds from water) and the generation of solar hydrogen fuel by photoelectrochemical water splitting. The first part of this work considers the concept of solar energy conversion by oxide semiconductors and the key performance-related properties, including electronic structure, charge transport, flat band potential and surface properties, which are responsible to the reactivity and photoreactivity of oxides with water. The performance of oxide systems for solar energy conversion is briefly considered in terms of an electronic factor. The progress of research in the formation of systems with high performance is considered in terms of specific aspects of nanotechnology, leading to the formation of systems with high performance. The nanotechnology approach in the development of highperformance photocatalysts is considered in terms of the effect of surface energy associated with the formation of nanostructured system on the formation of surface structures that exhibit outstanding properties. The unresolved problems that should be tackled in better understanding of the effect of nanostructures on properties and performance of oxide semiconductors in solar energy conversion are discussed. This part is summarised by a list of unresolved problems of crucial importance in the formation of systems with enhanced performance. This work also formulates the questions that must be addressed in order to overcome the hurdles in the formation of oxide semiconductors with high performance in water purification and the generation of solar fuel. The research strategy in the development of oxide systems with high performance, including photocatalysts for solar water purification and photoelectrodes for photoelectrochemical water splitting, is considered. The considerations are focused on the systems based on titanium dioxide of different defect disorder as well as its solid solutions and composites.
\end{abstract}

Keyword: Nanotechnology; Solar energy; Solar hydrogen fuel; Titanium dioxide; Water purification 